\title{
Evolving Solitons in Bubbly Flows
}

\author{
LEEN VAN WIJNGAARDEN \\ University of Twente, Enschede, The Netherlands
}

(Received: 12 September 1994)

\begin{abstract}
At the end of the sixties, it was shown that pressure waves in a bubbly liquid obey the $\mathrm{KdV}$ equation, the nonlinear term coming from convective acceleration and the dispersive term from volume oscillations of the bubbles.

For a variable $u$, proportional to $-p$, where $p$ denotes pressure, the appropriate $\mathrm{KdV}$ equation can be casted in the form $u_{t}-6 u u_{x}+u_{x x x}=0$. The theory of this equation predicts that, under certain conditions, solitons evolve from an initial profile $u(x, 0)$. In particular, it can be shown that the number $N$ of those solitons can be found from solving the eigenvalue problem $\psi_{x x}-u(x, 0) \psi=0$, with $\psi(0)=1$ and $\psi^{\prime}(0)=0 . N$ is found from counting the zeros of the solution of this equation between $x=0$ and $x=Q$, say, $Q$ being determined by the shape of $u(x, 0)$. We took as an initial pressure profile a shockwave, followed by an expansion wave. This can be realised in the laboratory and the problem, formulated above, can be solved exactly.

In this contribution the solution is outlined and it is shown from the experimental results that from the said initial disturbance, indeed solitons evolve in the predicated quantity.
\end{abstract}

Mathematics Subject Classification (1991): 58F07.

Key words: waves, solitons.

\section{Introduction}

In the 1960s and $1970 \mathrm{~s}$ it was recognised that it was not only gravity waves on the free surface of a liquid of finite depth that obeyed the Korteweg-de Vries (KdV) equation: other waves do, too.

Armed with experience, we can today safely predict that waves displaying both nonlinearity and dispersion are governed by a $\mathrm{KdV}$ equation, provided dissipation is sufficiently small. Pressure waves in bubbly fluids belong to this class, as was shown by Van Wijngaarden [11] who derived the corresponding $\mathrm{KdV}$ equation.

Experiments to verify this were carried out in Russia, as reported in the book by Nakoryakov et al. [5] and in our laboratory [6,7]. While there is no question that these experiments validated the theory qualitatively, it cannot be said that they did this in a completely quantitative way. Various reasons can be given for this. One of them is that many of these experiments concern transitions of the bore or shock-wave type, from one constant level of pressure to another. The Korteweg-de Vries equation has no steady solution for such a transition. In practice, seemingly steady, or quasi-steady pressure profiles are obtained due to an inevitable, though small, dissipation. Such a small dissipation is much less 
important when the disturbance, from a uniform quiescent state, is of finite spatial extent. Such experiments have, for example, been reported by Nakoryakov et al. [5]. However, a mathematical solution of the associated $\mathrm{KdV}$ equation was not given these. Also, as is emphasized by Watanabe and Prosperetti [8], thermal effects are extremely important in waves evolving over a long time, obscuring the dynamics.

With a view to these, rather recently gained, insights it seems worthwhile, and especially now that we are celebrating the 100th anniversary of the seminal paper by Korteweg and de Vries [3], to draw attention to a series of experiments which my student P. M. Roelofsen and I did about ten years ago, and which allow a comparison with the exact solution of the pertinent KdV equation.

\section{Description of Experiment}

The experiments discussed here were carried out in the facility described in detail by Noordzij and Van Wijngaarden [7]. The basic elements are shown in Figure 1.

A long tube of $11 \mathrm{~cm}$ diameter contains a mixture of water and small gas bubbles, mainly $\mathrm{CO}_{2}$ in these experiments. Above the free surface of the mixture, for times $t^{\prime}<0$ is a low pressure region with height $h_{1}$ and a high pressure region of height $h_{2}$.

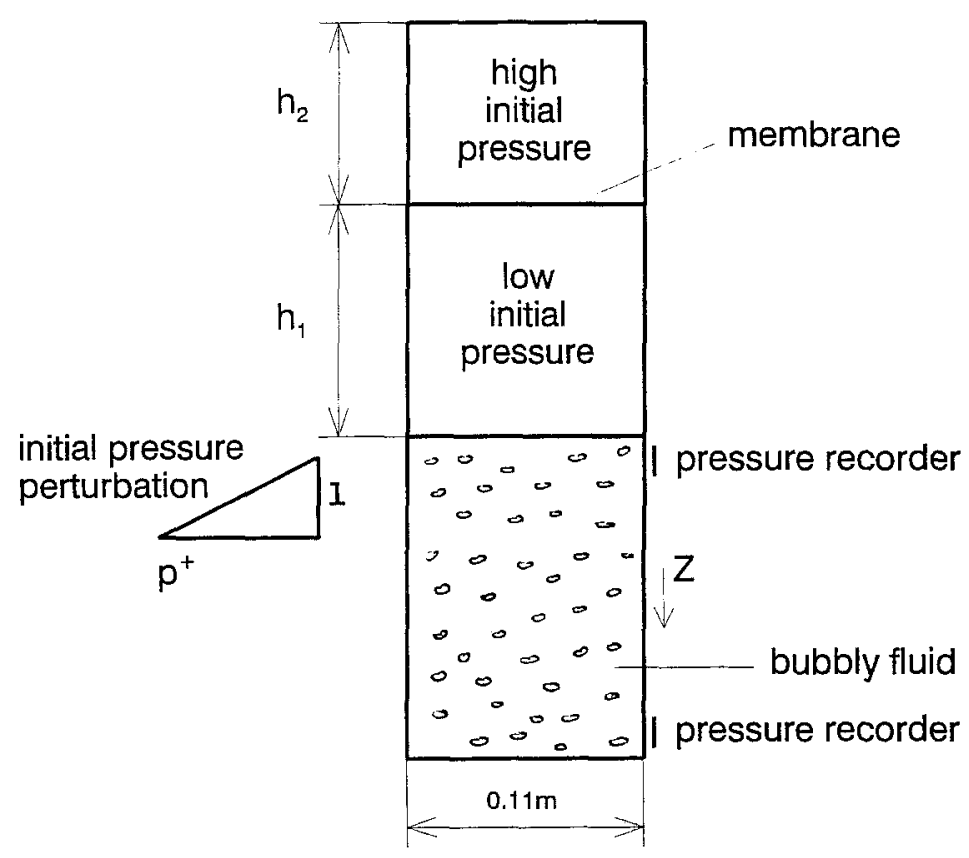

Fig. 1. Schematic representation of shock tube. 
Let the pressure for $t^{\prime}<0$ be $p_{0}$ in the low pressure region and in the mixture. At $t^{\prime}=0$ the membrane, which separates the low pressure region from the high pressure region, is punctured. By an adroit choice of $h_{1}, h_{2}$ and the pressure in the high pressure region a pressure perturbation is generated which, at the entrance to the mixture, $z=0$ in Figure 1, has the shape of a right-angled triangle.

First comes an instantaneous rise of the pressure to a value $p^{+}$, followed by an expansion wave in which the pressure linearly returns to $p_{0}$. Hence the perturbation is of finite extent, as opposed to a shock wave which produces a semi-infinite perturbation. Since the propagation into the bubbly water is both nonlinear (at sufficiently large $p^{+}-p_{0}$ ) and dispersive, the initial shape is not preserved but evolves according to the KdV equation.

Before passing on to the way in which this happens, we discuss the most important physical quantities in the experiments:

Bubble radius $a$, in the undisturbed conditions $a_{0}$. In our experiments these are all of the same radius, which varies from one experiment to the other, but is always of the order of $1 \mathrm{~mm}$.

Concentration of gas by volume $\alpha$. The initial value $\alpha_{0}$ can be measured from determining the static pressure distribution along the tube.

Velocity of sound $c_{0}$. Denoting the density of the fluid by $\rho$, the undisturbed pressure as before by $p_{0}$, the ratio of specific heats of the gas by $\gamma$ we have for this the relation [12]:

$$
c_{0}^{2}=\frac{\gamma p_{0}}{\rho \alpha_{0}\left(1-\alpha_{0}\right)} .
$$

Note that for values of $\alpha_{0}$ of a few percent, $c_{0}$ is of the order of $10^{2} \mathrm{~m} / \mathrm{s}$, under atmospheric conditions.

The natural frequency for small amplitude volume oscillations of a bubble $\omega_{B}$. This has been found by Minnaert [4] to be given by

$$
\omega_{B}^{2}=\frac{3 \gamma p_{0}}{\rho a_{0}^{2}} .
$$

At frequencies $\omega$ of external disturbances which are far below $\omega_{B}$, bubbles just follow the external pressure variations, and linear waves pass undeformed through the mixture with wavelength $2 \pi c_{0} / \omega$. The closer the frequencies approach $\omega_{B}$, the more this changes. The speed of propagation depends on $\omega$ for linear waves, long waves travelling faster than short waves. By nonlinear effects, the speed of propagation depends on the wave amplitude. The two phenomena together give rise to interesting behaviour. 


\section{Some Experimental Results}

At various places along the tube (Figure 1), pressure transducers are placed, enabling the recording of the pressure as a function of time. We have done experiments in which the pressure was recorded, generated by the initial signal, in $z=0$ in the form of a shock wave followed by an expansion wave.

Figures 2 and 3 show some results. In Figure 2 the pressure transducer is located at $47 \mathrm{~cm}$ from the free surface; in Figure 3 it is at $97 \mathrm{~cm}$. The particulars of the experiments are given in Table I. In Figure 2 both the profile in $z=0$ and the following profile in $z=0.47 \mathrm{~m}$ are shown; in Figure 3 only the profile in $z=0.97 \mathrm{~m}$. For this experiment $l=30 \mathrm{~cm}$ and $p^{+}-p_{0}=34 \mathrm{kPa}$. Clearly, in both experiments the original disturbance, in the shape of a right-angled triangle, breaks up into a distinct number of wave structures. This is precisely what solution of the KdV equation predicts. The outstanding feature of these experiments is that for this particular initial shape the number of evolving distinct structures, which we shall recognise as solutions, can be exactly calculated and compared with the experimental results.

\section{Theory}

We denote the dimensionless pressure perturbation by $v$,

$$
v=\frac{p-p_{0}}{p_{0}} .
$$

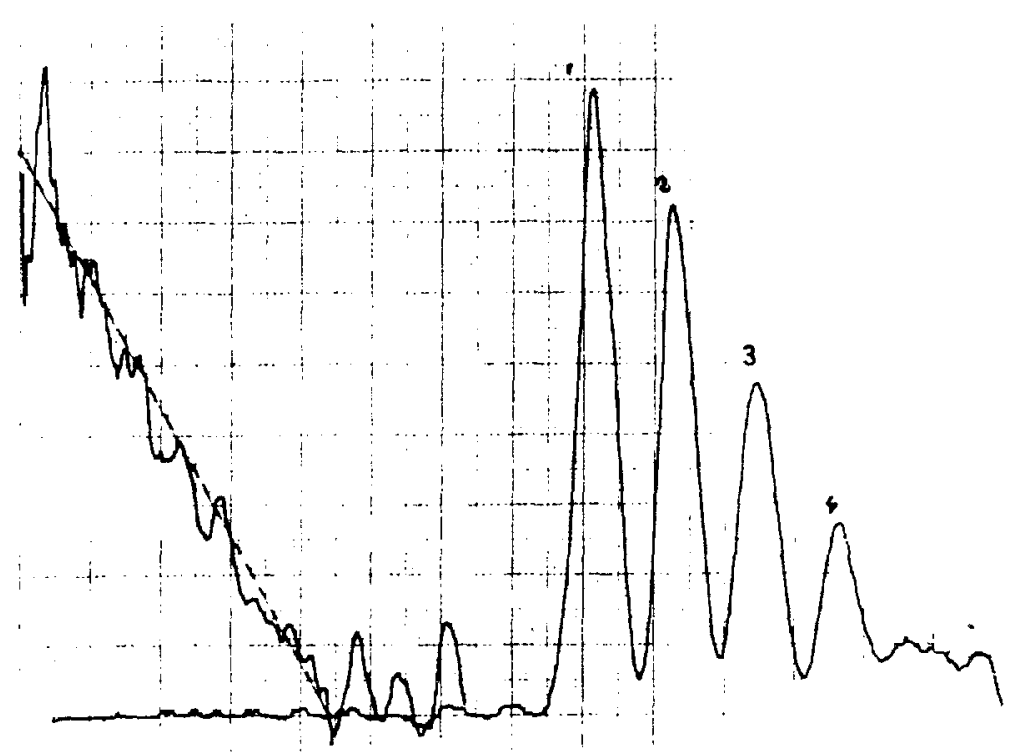

Fig. 2. The pressure profile on entering the mixture (left) and as it appears at the pressure transducer in $z=0.47 \mathrm{~m}$ below the interface. Data are given in Table I under 'exp 1'. 


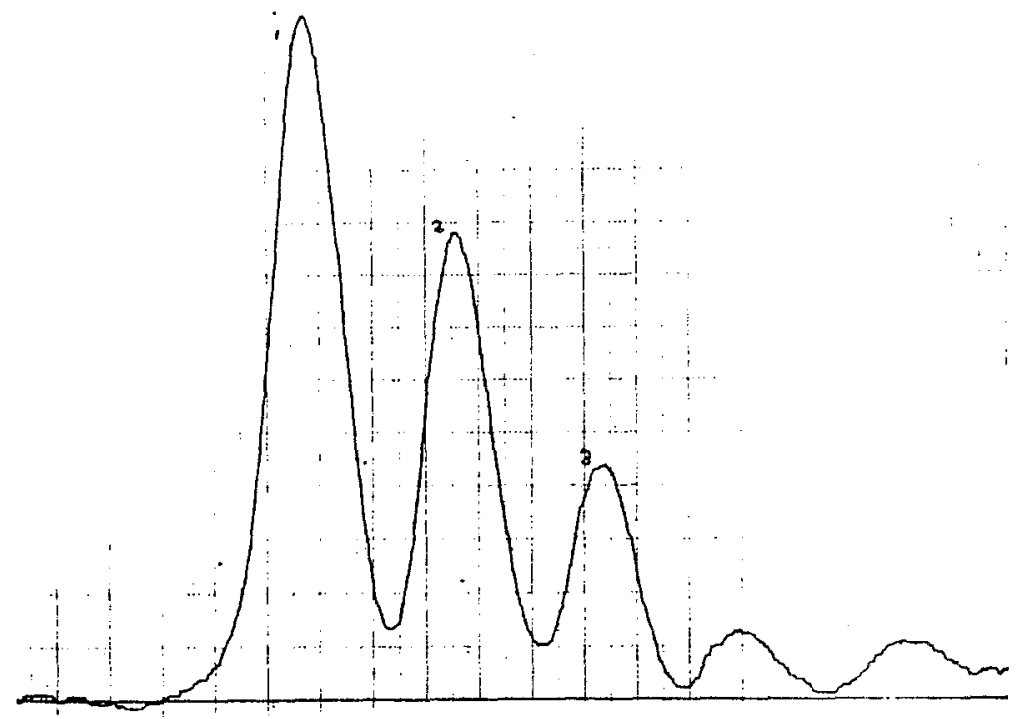

Fig. 3. Pressure profile measured by a transducer in $z=0.97 \mathrm{~m}$ below the interface following from the original profile with data as given in Table I under 'exp 4'.

Using the quantities defined in Section 2, and in addition the dispersion parameter $\sigma$,

$$
\sigma=\frac{a_{0}^{2}}{\alpha_{0}\left(1-\alpha_{0}\right)}=\frac{3 c_{0}^{2}}{\omega_{B}^{2}},
$$

Van Wijngaarden [11] showed that $v\left(z, t^{\prime}\right)$ evolves according to the equation

$$
\frac{\partial v}{\partial t^{\prime}}+c_{0} \frac{\partial v}{\partial z}+\frac{\gamma+1}{2 \gamma} c_{0} v \frac{\partial v}{\partial z}+\frac{\sigma c_{0}}{6} \frac{\partial^{3} v}{\partial z^{3}}=0
$$

It is convenient to choose a new coordinate $x^{\prime}=z-c_{0} t^{\prime}$, moving with the undisturbed speed of sound, and in addition the dimensionless variables $x, t$ and $\eta$, defined by

$$
\begin{aligned}
x^{\prime} & =\left\{\frac{\sigma l \gamma}{3(\gamma+1)}\right\}^{1 / 3} x, \\
t^{\prime} & =\frac{2 \gamma l}{(\gamma+1) c_{0}} t \\
v & =-\left\{\frac{72 \sigma \gamma}{l^{2}(\gamma+1)}\right\}^{1 / 3} u .
\end{aligned}
$$

We recall (see Figure 1) that $l$ is the length of the initial pressure disturbance. 
In terms of the variables $x, t$ and $u,(4.3)$ takes the standard form of the $\mathrm{KdV}$ equation employed in the literature (e.g., [9, p. 586])

$$
\frac{\partial u}{\partial t}-6 u \frac{\partial u}{\partial x}+\frac{\partial^{3} u}{\partial x^{3}}=0
$$

This equation is valid according to the theory, provided:

(i) dispersion is weak;

(ii) dissipation is negligible; and

(iii) nonlinearity is weak.

The first condition, (i), means that a typical frequency $c_{0} / l$ must be small with respect to $\omega_{B}$. The ratio $c_{0} / \omega_{B} l$ is about 0.1 in our experiments. The second condition, (ii), is also fulfilled. When we take dissipation (viscous, but mainly thermal) into account, this would in Equation (4.7) lead to an additional term $0.06 \partial^{2} u / \partial x^{2}$ on the left-hand side. Such a term is negligible in our case, where the scale is determined by (4.4) but becomes important for phenomena of large extent. In other words, the approximation underlying (4.7) is not a uniformly valid one. Regarding (iii) we have chosen pressure disturbances of moderate value, $0.2-0.5$, guaranteeing a not-too-strong nonlinearity.

Theory predicts that out of the initial profile $u(x, 0)$ evolve

(a) $N$ solitons, $N$ to be found from inverse scattering theory, and travelling in the positive $x$ direction;

(b) a continuous wave train travelling in the negative $x$ direction.

We recall that the $x$-axis moves with speed $c_{0}$ in the $z$ direction. This means that the continuous wave train moves in the laboratory frame rapidly in the negative $z$ direction. These waves can easily be distinguished from the solitons, since they have small amplitude and move quickly. We shall therefore restrict our attention here to the solitons. These emerge from the spectrum of discrete eigenvalues of the Schrödinger equation

$$
\frac{\mathrm{d}^{2} \varphi}{\mathrm{d} x^{2}}+\{\lambda-u(x, t)\} \varphi=0
$$

in which $u$ serves as potential through Miura's transformation

$$
u=\frac{1}{\varphi} \frac{\mathrm{d}^{2} \varphi}{\mathrm{d} x^{2}}+\lambda
$$

The eigenvalues $\lambda_{n}$ of (4.8) are negative, $\lambda_{n}=-k_{n}^{2}$, and, most importantly, independent of time. They are ordered such that $k_{1}>k_{2}>k_{3}>\cdots>k_{N}>0$, where $N$ is finite. Because they are independent of time, they can be found from solution of

$$
\frac{\mathrm{d}^{2} \varphi}{\mathrm{d} x^{2}}+\{\lambda-u(x, 0)\} \varphi=0 .
$$


Asymptotically - that is for large $x$ and $t$ - each eigenvalue corresponds to a soliton. The problem is therefore to find the total number, $N$, of eigenvalues. In the present problem, this can be found exactly.

First we insert the appropriate form of $u(x, 0)$ into (4.10). In physical coordinates $x^{\prime}$ or $z$ (which are the same at $t^{\prime}=0$ ), the pressure profile is

$$
\begin{aligned}
v=\frac{p-p_{0}}{p_{0}} & =\frac{p^{+}-p_{0}}{p_{0}} \frac{x^{\prime}}{l}, \quad 0<x^{\prime}<l \\
& =0, \quad x^{\prime} \geqslant l \quad \text { and } \quad x^{\prime} \leqslant 0 .
\end{aligned}
$$

Using (4.4) and (4.6) this gives for $u(x, 0)$

$$
\begin{aligned}
u(x, 0) & =-\frac{p^{+}-p_{0}}{p_{0}} \frac{x}{6}, \quad x<\left\{\frac{3 l^{2}(\gamma+1)}{\gamma \sigma}\right\}^{2 / 3} \\
& =0, \quad x \leqslant 0 \quad \text { and } \quad x \geqslant\left\{\frac{3 l^{2}(\gamma+1)}{\gamma \sigma}\right\}^{2 / 3} .
\end{aligned}
$$

We consider solutions of (4.10), $u(x, 0)$ being given by (4.12), and with boundary conditions

$$
x=0: \frac{\partial \varphi}{\partial x}=0, \quad \varphi=1 .
$$

Sturm's First Comparison Theorem (see, e.g., [2, Ch. 10]) states the following: suppose a solution, $\varphi_{a}$, corresponding with $\lambda_{a}$, has two zeros, in $x_{1}$ and $x_{2}$, say, on the $x$-axis. Then a solution $\varphi_{b}$, for $\lambda_{b}>\lambda_{a}$, has an additional zero in between $x_{1}$ and $x_{2}$. Now we apply this to our problem. For $\lambda \rightarrow-\infty$ the solution of (4.10), subjected to (4.13), is $\cosh \lambda x$ which has no zeros for real $x$. Hence the solution $\varphi_{1}$ corresponding with the largest value of $k, k_{1}$, has one zero on the $x$ axis, the solution $\varphi_{2}$ associated with $k_{2}$ has two zeros, etc. Since all eigenvalues are negative, the solution corresponding with $\lambda=0$ has as many zeros as the one with the nearest eigenvalue which is $\lambda_{N}=-k_{N}^{2}$. Therefore, we have to determine the number of zeros of the solution of (4.10) with $\lambda=0$ and $u(x, 0)$ given by (4.12) and with (4.13) as boundary conditions. (This method has been used before by Hammack and Segur [1] in a problem concerning waterwaves.)

We take $\lambda=0$ and insert (4.12) into (4.10). It is convenient to use instead of $x$ a new coordinate $y$, given by

$$
x=\left(\frac{6 p_{0}}{p^{+}-p_{0}}\right)^{1 / 3} y .
$$

Upon using this in inserting (4.12) into (4.10), the latter equation is transformed into the Airy equation [10],

$$
\frac{\mathrm{d}^{2} \varphi}{\mathrm{d} y^{2}}+y \varphi=0, \quad 0<y<Q
$$




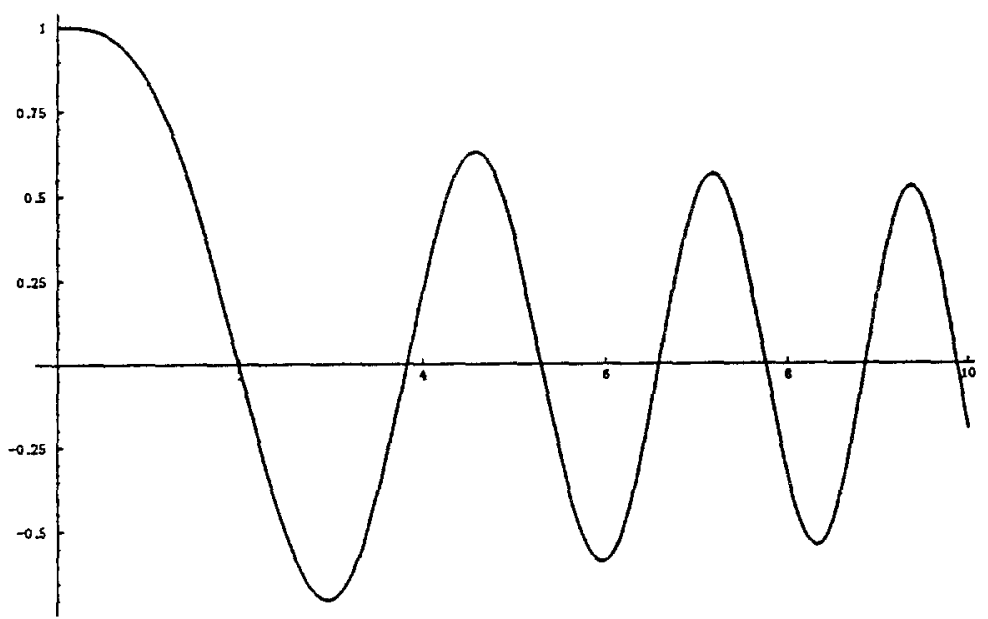

Fig. 4. The function $1.408 A i(-y)+0.813 B i(-y)$, in the right-hand side of (4.20).

where

$$
Q=\left\{\frac{p^{+}-p_{0}}{p_{0}} \frac{(\gamma+1) l^{2}}{2 \sigma \gamma}\right\}^{1 / 3}
$$

and

$$
\frac{\mathrm{d}^{2} \varphi}{\mathrm{d} y^{2}}=0, \quad y \leqslant 0 \quad \text { and } \quad y \geqslant Q
$$

The boundary conditions (4.13) become

$$
y=0: \frac{\partial \varphi}{\partial y}=0, \quad \varphi=1 .
$$

The system (4.15)-(4.18) has the solution

$$
\begin{aligned}
& y<0, \quad \varphi=1 \\
& 0 \leqslant y \leqslant Q, \quad \varphi=1.408 A i(-y)+0.813 B i(-y), \\
& y>Q, \quad \vec{\varphi}=\varphi=\left(\frac{\mathrm{d} \varphi}{\mathrm{d} y}\right)_{y=Q}(y-Q)+\varphi(Q),
\end{aligned}
$$

$\varphi$ being given by (4.20).

Figure 4 shows a sketch of the function on the right-hand side of (4.20). For a given value of $Q$ we have to count the zeros in $0<y<Q$. The part in $y<0$ 
has no zeros, whereas the part of the solution in $y>Q$ contributes an additional zero when

$$
\left\{\frac{\mathrm{d} \varphi}{\mathrm{d} y} / \varphi\right\}_{y=Q}<0
$$

\section{Comparison with Experiment}

In Table I, we collect data of a number of our experiments. In the final columns the number $N_{t}$ of solitons is given, as following from the theory, expounded above, and the number $N_{e}$ from the corresponding experiment.

From the table it follows that in all experiments the number of solitons in both theory and experiment agree completely. The experiments shown in Figures 2 and 3 show this for experiments 1 and 4 in the table, respectively. The same agreement holds also for other experiments, not displayed here, verifying the validity of the $\mathrm{KdV}$ equation for these waves.

\section{Shape of Solitons}

We have labelled the evolving structures somewhat loosely as solitons. In fact, the distinct pressure humps shown in Figures 2 and 3 are not yet solitons, because they are still interacting and will only further down the tube become true solitons. If the peak pressure in a soliton is $p_{s}$, and accordingly $v_{s}=\left(p_{s}-p_{0}\right) / p_{0}$, a soliton has the shape

$$
v=v_{s} \operatorname{sech}^{2}\left[\left(\frac{v_{s}}{2} \frac{\gamma+1}{\sigma \gamma}\right)^{1 / 2}\left(x^{\prime}-\frac{1}{3} \frac{\gamma+1}{2 \gamma} v_{s} t^{\prime}\right)\right]
$$

In some experiments, with the pressure transducer at $z=0.97 \mathrm{~m}$ or further down, this shape could indeed be verified. At closer distance solitons are still undergoing evolution to their final shape. Their number, however, does not change, anymore.

\begin{tabular}{|c|c|c|c|c|c|c|c|c|}
\hline Exp. & $\begin{array}{l}\text { Location } \\
\text { press. transd. } \\
\mathrm{m}\end{array}$ & $\begin{array}{l}\text { Void } \\
\text { fraction } \alpha \\
\%\end{array}$ & $\mathrm{~m}$ & $\begin{array}{l}C_{0} \\
\mathrm{~m} \mathrm{~s}^{-1}\end{array}$ & $\frac{p^{+}-p_{0}}{p_{0}}$ & $Q$ & $N_{t}$ & $N_{e}$ \\
\hline 1 & 0.47 & 0.74 & 0.24 & 107 & 0.52 & 6.6 & 4 & 4 \\
\hline 2 & 0.47 & 0.74 & 0.19 & 107 & 0.30 & 4.7 & 3 & 3 \\
\hline 3 & 0.47 & 0.74 & 0.16 & 107 & 0.16 & 3.4 & 2 & 2 \\
\hline 4 & 0.97 & 0.43 & 0.30 & 157 & 0.34 & 5.8 & 3 & 3 \\
\hline 5 & 0.97 & 1.32 & 0.13 & 90 & 0.13 & 2.7 & 1 & 1 \\
\hline 6 & 0.97 & 0.74 & 0.18 & 118 & 0.25 & 4.3 & 2 & 2 \\
\hline
\end{tabular}

TABLE I. 


\section{References}

1. Hammack, J. L. and Segur, H.: The Korteweg-de Vries equation and water waves. Part 2, Comparison with experiments, J. Fluid Mech. 65(2), (1974), 289-314.

2. Ince, E. L.: Ordinary Differential Equations, Dover Publications, 1956.

3. Korteweg, D. J. and de Vries, G.: On the change of form of long waves advancing in a rectangular canal and on a new type of long stationary waves, Phil. Mag. 39(240) (1895), 422.

4. Minnaert, M.: Musical air bubbles and the sound of running water, Phil. Mag. 16 (1933), 235.

5. Nakoryakov, V. E., Pokusaev, B. C., and Shreiber, I. R.: Wave Propagation in Gas-Liquid Media, Ch. 3, 1993.

6. Noordzij, L.: Shock waves in bubble-liquid mixtures, in L. I. Sedov (ed.), Non-Steady Flow of Water at High Speeds, Proc. IUTAM Symp., Nauka, Moscow, 1973.

7. Noordzij, L. and Wijngaarden, L. van: Relaxation effects, caused by relative motion, on shock waves in gas-bubble/liquid mixtures, J. Fluid Mech. 66(1) (1974), 115-143.

8. Watanabe, M. and Prosperetti, A.: Shock waves in dilute bubbly liquids, J. Fluid Mech. 274 (1994), 349-381.

9. Whitham, G. B.: Linear and Nonlinear Waves, Wiley, New York, 1974.

10. Whittaker, E. T. and Watson, G. N.: A Course of Modern Analysis, Cambridge University Press, Cambridge, 1952.

11. Wijngaarden, L. van: On the equations of motion for mixtures of liquid and gas bubbles, $J$. Fluid Mech. 33(3) (1968), 465-474.

12. Wijngaarden, L. van: One-dimensional flow of liquids containing small gas bubbles, Ann. Rev. Fluid Mech. 4 (1972), 369-396. 